\title{
Wearable Functional Near-Infrared (FNIR) Technology and Its Applications in Naturalistic Conditions
}

\author{
Francis Tsow and Audrey Bowden* \\ Department of Biomedical Engineering, Vanderbilt University, USA \\ *Corresponding author: Audrey Bowden, Department of Biomedical Engineering, Vanderbilt University, USA. \\ To Cite This Article: Audrey Bowden. Wearable Functional Near-Infrared (FNIR) Technology and Its Applications in Naturalistic Conditions. Am J \\ Biomed Sci \& Res. 2019 - 5(1). AJBSR.MS.ID.000869. DOI: 10.34297/AJBSR.2019.05.000869
}

Received: 韭 August 26, 2019; Published: 䟧 September 03, 2019

\begin{abstract}
Wearable functional near-infrared technology (fNIR) is a noninvasive neuroimaging technology that is affordable, low cost, and user-friendly, making it a promising technology to study how our brain works in naturalistic conditions. A practical user-friendly fNIR device that allows naturalistic use (e.g., home-use) can open unprecedented opportunities with health, psychologic, political/economic, and physiologic impacts. Wearable fNIR devices impose additional requirements compared to laboratory-based fNIR systems, including size, weight, and motion/ambient artifacts, without sacrificing key performance. Furthermore, to ensure user-friendliness, special attentions to cost, power, connectivity, flexibility to adapt to various applications, and comfort have to be properly considered. This manuscript reviews wearable fNIR in naturalistic conditions, covering reported applications and technologies developed to enable fNIR wearability. We also highlight some of the remaining challenges of current wearable fNIR devices including the limited number of optodes, uncorrected artifacts and noise, and the need for user alignment and manual event recording.
\end{abstract}

Keywords: FNIR; functional near-infrared; Wearable; Recognitive; Brain Hemodynamics; Oxygenation; Naturalistic condition

Abbreviations: FNIR: functional Near-Infrared; EEG: Electroencephalogram; TMS: Transcranial Magnetic Stimulation; PET: Positron Emission Tomography; FMRI: Functional Magnetic Resonance Imaging; MRI: Magnetic Resonance Imaging; ECG: Electrocardiogram

\section{Introduction}

Understanding how our brain works in a tangible way has tremendous health, psychologic, physiologic, political, and economic impact. Consequently, functional neuroimaging has fostered intense interest in the development of tools that feature varying degrees of cost, accuracy, usability, spatial and temporal resolutions [1]. One of the most common tools of functional neuroimaging, electroencephalography (EEG), is a technology that measures electrical signals from cortical neurons with microsecond resolution. However, its data are limited to the surface of the brain. Functional magnetic resonance imaging (fMRI) and positron emission tomography (PET) have also been used in many cognitive studies involving brain function or activity. However, current systems are bulky, slow and require the subjects to be immobilized to reduce motion artifacts. Furthermore, PET typically uses a radioactive tracer, which limits its use in naturalistic conditions (i.e., natural, everyday conditions). In contrast, fNIR (functional nearinfrared spectroscopy) offers a relatively fast response, reasonable spatial resolution and more tolerance to motion [2,3] compared to PET, ECG, and MRI. Just as importantly, fNIR can be readily implemented in a competitively smaller form factor, at lower cost, and with minimal invasiveness. These advantages allow fNIR to be potentially deployed in naturalistic conditions as a wearable device.
This review aims to provide a background on wearable functional near-infrared (fNIR) technology, its current status, and its potential applications in naturalistic conditions.

FNIR spectroscopy uses infrared light to probe the hemodynamics of brain tissue, which in turn reflects the metabolism of the brain and how it functions [4]. FNIR is based on the differences in absorbance or scattering of oxyhemoglobin and deoxyhemoglobin in cerebral blood at two different wavelengths. With signals from additional wavelengths, metabolism (oxidized cytochrome-c-oxidase) can also be probed [5]. Jobsis was the first to demonstrate that it is possible to detect hemodynamics in adult cortex during hyperventilation [4]; later, the first fNIR studies were conducted by Chance et al. with one channel in 1992 and with multiple simultaneous channels by Hoshi et al. in 1993 [6,7]. Common fNIR detection modes include continuous wave, frequency domain, and time domain, with continuous wave being the simplest to implement and the least expensive, although it provides changes in concentrations of oxy- and deoxy-hemoglobin rather than absolute values [8]. Many wearable fNIR devices have adopted the continuous wave mode in the interest of a compact and low-cost design, which will have a direct effect on its user friendliness and affordability. 
Traditionally, many of the mental tasks designed for fNIR cognitive tests were performed in clinical settings, which pose environmental and confounding factors that are different from naturalistic conditions and do not reflect our everyday events [9]. To enable cognitive tests in naturalistic conditions, several groups have developed wearable fNIR systems [8]. For example, Almajidy et al. developed a multichannel NIRS system [10]. Piper et al. later developed a wearable multi-channel fNIRS system for freely moving subjects using a head cap [11]. Strangman et al. developed a wearable device with a multimodal physiological monitor with leads for biopotentials; 64 NIRS optodes (a typical optode consists of a light source and detector pair); force, acceleration, gyroscope, and temperature sensors; and the ability to connect to a commercial respirometer [12]. A wearable fNIRS-EEG system with 128 channels for fNIRS, 32 channels for EEG, two channels for auxiliary electrocardiogram (ECG) and an accelerometer for correction in the form of a cap was developed by Kassab et al [13]. However, despite the technological advances of wearable fNIR technology, the use of a cap to fix the optodes on the subject makes it uncomfortable for prolonged use [14] (e.g., everyday home use). As an alternative design, application of fNIR in a naturalistic environment with a fiber-less, multichannel wearable device in the form of a headset connected to a portable processing unit was demonstrated by Pinti et al [15]. Wyser et al. later developed a modular wearable device with a flexible form factor allowing multiple distances between optode source and detectors; it also featured multiple wavelengths [16]. Wyser's optode modules are about the size of a quarter and allows the use of headgear other than a cap; however, there is still a somewhat bulky control unit.

Besides wearable fNIR devices developed by research groups, there are also a number of commercially available fNIR devices in the market, including Biopac Systems Inc., Artinis Medical Systems (https://www.artinis.com/), fNIR Devices (http://fnirdevices. com/), and NIRX (https://nirx.net/). While many of them are highly flexible, accurate and convenient to use in a laboratory setting, they tend to be costly (from a few thousands to tens of thousands of dollars) and relatively large, making the use of these devices challenging to carry out population studies.

Advances in wearable fNIR [5] allow new studies in cognitive science, social science, physiology (specifically in neurodevelopment such as attention deficit/hyperactivity disorder or autism spectrum disorder) [5], psychology, brain-machine interfaces, medical screening (such as stroke) [17-19] and many other areas [6-20]. A plethora of interesting applications for fNIR have been demonstrated in recent years, including fNIR responses of professional violinists playing duets [21], actors acting together [22], subjects anticipating a mentally effortful task [23], subjects engaging in problem solving and spatial navigation [24-26], subjects responding to handheld and virtual reality navigation devices [27], patients' cerebral hemodynamics response to anesthetics [28], partners' neural responses and synchronization during eye-to-eye contact [29]. In addition, wearable fNIR has also demonstrated capability in identifying patients with Pakinsonian syndromes having higher prefrontal cortex activity to maintain posture stability [30] and subjects with traumatic brain injury [31]. Brain-computer interface [32] and correlation with fNIR hybrid system [33] have also been demonstrated. In summary, wearable fNIR allows unprecedented studies and diagnosis. However, many of these studies have been confined to laboratories or clinics (except campus navigation using wearable fNIR [27]), rather than in naturalistic conditions (i.e., homes).

\section{Methods}

Given the promises, challenges, and advances of wearable fNIR technologies, a number of excellent reviews on wearable fNIR technologies deployable in naturalistic environments have been published [5,20,34]. We have performed a systematic search on recent advances and summarize below the various applications that have been published using wearable fNIR technologies in naturalistic environment as well as the features that have enabled fNIR technologies to move from the laboratory to naturalistic environments. Our search was based on entering the keywords "wearable fNIR," "functional near-infrared natural environment," and "fNIR natural" in Web of Science to search for relevant publications in this topic. A total of 30 matches was found with 20 unique and relevant publications from 1900-2019.

\section{Results}

\section{Wearable fNIR Applications}

(Table 1) provides a summary of different applications of fNIR to naturalistic environments. We grouped the results into four categories on the basis of field of applications: namely, health, psychologic, political/economic, and physiologic applications. Under each broader category, there can also be sub-categories. For instance, under health, there are sub-categories pertaining to monitoring/screening and prevention applications. Example applications under these subcategories include application of fNIR to the monitoring of preterm infant brain health (hypoxia) [35], neural activities during cardiac and vascular surgery, cerebrovascular disease, epilepsy, and headache [36]. In addition, fNIR can also be used to predict likelihood of obesity and potentially used to prevent obesity [37]. Furthermore, it can be used to study decision-making, which can find applications in marketing, advertising, learning and training optimization by understanding demands placed on individuals as they engage with various tasks [5]. By capturing cognitive capacity/limit (i.e., maximum brain activity to maintain performance), operator efficiency can be maximized while human error can be minimized [5,38]. To study social interactions, a technique called hyperscanning has been employed. Hyperscanning refers to simultaneous scanning of the brains of interacting subjects. It is a way to study social interaction and neural functions and has been demonstrated in prefrontal cortex activation during acting and other activities [4]. Combining 
virtual reality and fNIR allows control of subject's experience in dynamic environment [5]. fNIR can also serve as a research tool for studying brain activation in various activities, decision-making and cognitive states where selective attention and physical work compete with executive processing (e.g., balancing a ping-pong ball on a small card while walking) [38,39]. It has also been applied to distinguish which part of a story the subject was listening and to understand the neural mechanism underlying imagined rather than intelligible speech for communication $[40,41]$ (Table 1).

\begin{tabular}{|c|c|c|}
\hline Categories & Sub-categories & Applications \\
\hline \multirow{5}{*}{ Health } & \multirow{4}{*}{ Monitoring/Screening [36] } & Cardiac and vascular surgery Neonatology \\
\hline & & Neonatology \\
\hline & & Cerebrovascular disease \\
\hline & & Epilepsy and headache \\
\hline & Prevention [37] & Obesity Prediction \\
\hline \multirow{6}{*}{ Psychologic } & \multirow{4}{*}{ Performance [34] } & Cognitive workload \\
\hline & & Cognitive capacity \\
\hline & & Efficiency improvement \\
\hline & & Error minimization \\
\hline & \multirow{2}{*}{ Behavior $[5,41]$} & Social interaction \\
\hline & & Listened speech recognition \\
\hline \multirow{3}{*}{ Political/economic } & \multirow{2}{*}{ Decision-making [5] } & Decision-making (marketing) \\
\hline & & Decision making (advertising) \\
\hline & Product development [40] & Unintelligible speech recognition \\
\hline \multirow{6}{*}{ Physiologic } & \multirow{6}{*}{ Brain studies $[5,22,38,39]$} & Acting \\
\hline & & Control of subject's experience \\
\hline & & Driving \\
\hline & & Open-ended situations \\
\hline & & Multitasking \\
\hline & & Social interaction \\
\hline
\end{tabular}

\section{Wearable fNIR requirements}

A wearable fNIR device has additional requirements compared to a laboratory-based fNIR system. In a laboratory, the subjects are more stationary and the environment is well controlled. If the subjects are engaged in activities, more motion can be expected. Due to the sensitivity of the optical detection module of the fNIR device, unaccounted motions will manifest as noise. In general. the heavier the unit, the more it tends to move due to inertia, resulting in more motion artifacts (i.e., noise) and adding stress on the connecting wires/cables. To reduce motion artifacts, attempts have been made to apply correction algorithms [42], to incorporate motion sensors such as an accelerometers and gyroscopes and to optimize the size and mass of the optodes [2]. Several groups have studied the effects of the distance between the source and detector of an optode [10], and some have employed a short channel $(5 \mathrm{~mm}$ to $1 \mathrm{~cm})[16,43]$ to correct for artifacts due to superficial tissue and motion. Wireless designs have also been implemented to eliminate connecting wires/ cables $[20,31,39]$ to enhance comfort and to remove restriction to subjects' activities.

\begin{tabular}{|c|c|}
\hline Key features for wearability & Justification \\
\hline Low cost $[5,9,12]$ & Enable better portability (i.e., smaller, more affordable by replacing costly computer with onboard processing) \\
\hline Cable-free $[20,31,39]$ & Allow naturalistic measurements with wireless connectivity (e.g., ambulatory tasks) \\
\hline $\begin{array}{l}\text { Compact and light-weight } \\
\qquad[20,44]\end{array}$ & $\begin{array}{l}\text { Leverage advancement in electronics, optics, and computing for fNIR miniaturization and integration (i.e., a smaller } \\
\text { and lighter device allows natural physical activities) }\end{array}$ \\
\hline Comfort $[5,15,24]$ & $\begin{array}{l}\text { Allow comfortable, fast, flexible, and accurate placement of multiple optodes (e.g., headband with optode matching } \\
\text { holes) }\end{array}$ \\
\hline Noise reduction $[5,42,45]$ & $\begin{array}{c}\text { Reduce motion artifacts due to subjects moving in naturalistic environments (e.g., applying algorithms with and } \\
\text { without additional sensors) }\end{array}$ \\
\hline Flexibility $[1,5,16,44]$ & $\begin{array}{l}\text { Improve debugging, scalability, reusability, flexibility and potential disposability for improved reliability and } \\
\text { reproducibility using modular design. }\end{array}$ \\
\hline
\end{tabular}


Furthermore, many wearable fNIR devices adopt the use of a cap to fix the optodes in place, although prolonged use of the cap can result in discomfort. A smaller and more flexible form factor (e.g., a headband) can provide comfort to the user and stability to the optodes $[5,15,24]$.

Finally, a modular design can streamline the number of components to be mounted on the subject, thus reducing the overall weight, size, and cost of the wearable fNIR device. Furthermore, the use of a modular design can allow parts to be easily switched out, thus affording the use of disposable components for hygiene or performance reasons $[1,5,16,44]$. We have summarized key features from the searched literatures implemented by wearable fNIR devices (Table 2).

Although there has been tremendous technical progress in wearable fNIR devices, there are still remaining challenges that needs to be addressed for a truly user-friendly, home-use, wearable system. While there are existing algorithms and sensors that can help correct for motion artifacts, the correction still has room to improve. Furthermore, in order to capture as much neural activity as possible, an fNIR device should have wide coverage over different brain regions. As a result, multiple optodes will have to spread over the entire surface of the head where the brain resides. Increasing the number of optodes is often difficult for a low cost, low power fNIR device, as each additional optode adds cost and power consumption to the device. To reduce the required number of optodes, optode alignment will be critical. A way to help align different brain regions for different targeted functions, including alignment with dorsolateral prefrontal cortex for working memory and attention, pars opercularis for language production and motor cortices for articulation, would be helpful in accurately assessing cognitive and behavior functions [29,46]. In addition, hair can often get in the way of obtaining high quality fNIR signal. In order to probe areas of the scalp where hair might be present, a brush-like structure that allows hair to be pushed aside has been demonstrated by Khan et al. [3]. Some groups have also taken the approach of focusing only on the prefrontal cortex (PFC), which has been shown to be critical to working memory [47], avoiding the issue of hair altogether. Nonetheless, measuring only the prefrontal cortex will miss out on other important regions such as the motor cortex [3].

\section{Conclusion}

A cost-effective neuroimaging tool such as an fNIR device can potentially lead to novel cognitive and social research, non-invasive brain-machine interfaces and new options for point-of-care screening/diagnosis. Wearable fNIR devices are becoming more user-friendly and more reliable. Some groups have already started adding other modalities (such as electroencephalogram (EEG) or Transcranial magnetic stimulation (TMS)) to further augment the functionality of the wearable fNIR device. To be truly ready for largescale home use, remaining challenges include better correcting of artifacts and noise, packing more optodes into the same compact and low-cost form factor, and removing the need for user alignment. In the future, new and novel sensor technologies for higher signalto-noise ratio, high-density sensor array, automatic alignment of brain regions, and autonomous recording of triggering events will help enable research that is currently tedious or difficult to perform. For instance, a Bluetooth ${ }^{\circledR}$ can serve as a proximity sensor to sense interaction and an acoustic device can be used for voice capturing of events. A number of tools and research efforts have focused on assisting researchers in finding the right brain region to study [46, 48-53]. A topological mapping ability of the fNIR device can help address this challenge independent of the availability of MRI images.

Currently, wearable fNIR devices have a limited number of optodes. The number is typically fixed at the design stage, as each optode is hardwired to the ADC converter. To make the design more flexible, optodes can be wirelessly connected to the ADC converter. To reduce power and cost for the wireless implementation, RFID or acoustic approaches can be employed. Furthermore, data from various channels should be multiplexed to further save on power and cost. Frequency-division multiplexing, time-division multiplexing, or code-division multiplexing can be used. We anticipate a user-friendly, low-cost, wearable fNIR device would allow many important social/behavioral/psychological questions to be answered. Further, we expect the results will lead to important contributions to behavior science, health care and engineering: for example, in understanding the recognitive role in obesity and in child development, enhancing medical diagnosis, creating novel brain-machine interface, and developing new marketing and advertising tools.

\section{Acknowledgements}

This research project was sponsored by the Dorothy J Wingfield Phillips Chancellor Faculty Fellowship.

\section{Conflict of Interest}

No conflicts of interest.

\section{References}

1. Izzetoglu K, Bunce S, Izzetoglu M, Onaral B, Pourrezaei K (2004) Functional near-infrared neuroimaging. Conf Proc IEEE Eng Med Biol Soc 7: 5333-5336.

2. Zhang Q Yan X, Strangman GE (2011) Development of motion resistant instrumentation for ambulatory near-infrared spectroscopy. J Biomed Opt 16(8): 87008.

3. Khan B, Wildey C, Francis R, Tian F, Delgado MR, et al. (2012) Improving optical contact for functional near-infrared brain spectroscopy and imaging with brush optodes. Biomed Opt Express 3(5): 878-898.

4. Jobsis FF (1977) Noninvasive, infrared monitoring of cerebral and myocardial oxygen sufficiency and circulatory parameters. Science 198(4323): 1264-1267.

5. Pinti P, Aichelburg C, Gilbert S, Hamilton A, Hirsch J, et al. (2018) A Review on the Use of Wearable Functional Near-Infrared Spectroscopy in Naturalistic Environments. Jpn Psychol Res 60(4): 347-373. 
6. Ferrari M, Quaresima V (2012) A brief review on the history of human functional near-infrared spectroscopy (fNIRS) development and fields of application. Neuroimage 63(2): 921-935.

7. Chance B, Zhuang Z, UnAh C, Alter C, Lipton L (1993) Cognition-activated low-frequency modulation of light absorption in human brain. Proc Natl Acad Sci U S A 90(8): 3770-3774.

8. Scholkmann F, Kleiser S, Metz AJ, Zimmermann R, Mata Pavia J, et al. (2014) A review on continuous wave functional near-infrared spectroscopy and imaging instrumentation and methodology. Neuroimage 85 Pt 1: 6-27.

9. Si J, Zhao R, Zhang Y, Zuo N, Zhang X, et al. A portable fNIRS system with eight channels. In: Hirschberg H, et al. [Eds]. (2015) Optical Techniques in Neurosurgery, Neurophotonics, and Optogenetics II. Proceedings of SPIE 9305.

10. Almajidy RK, Hofmann UG. On the design of a multi-channel NIR system to monitor functional brain activity. NIR2013 Proc. 2013;(March 2014):335-338.

11. Piper SK, Krueger A, Koch SP, Mehnert J, Habermehl C, et al. (2014) A wearable multi-channel fNIRS system for brain imaging in freely moving subjects. Neuroimage 85 Pt 1: 64-71.

12. Strangman GE, Ivkovic V, Zhang Q (1985) Wearable brain imaging with multimodal physiological monitoring. J Appl Physiol 124(3): 564-572.

13. Kassab A, Le Lan J, Tremblay J, Vannasing P, Dehbozorgi M, et al. (2018) Multichannel wearable fNIRS-EEG system for long-term clinical monitoring. Hum Brain Mapp 39(1): 7-23.

14. Kassab A, Le Lan J, Vannasing P, Sawan M (2015) Functional nearinfrared spectroscopy caps for brain activity monitoring: a review. Appl Opt 54(3): 576-586.

15. Pinti P, Aichelburg C, Lind F, Power S, Swingler E, et al. (2015) Using Fiberless, Wearable fNIRS to Monitor Brain Activity in Real-world Cognitive Tasks. J Vis Exp (106): 1-13.

16. Wyser D, Lambercy O, Scholkmann F, Wolf M, Gassert R (2017) Wearable and modular functional near-infrared spectroscopy instrument with multidistance measurements at four wavelengths. Neurophotonics 4(4): 041413.

17. Aries MJ, Coumou AD, Elting JW, van der Harst JJ, Kremer BP, et al (2012) Near Infrared Spectroscopy for the Detection of Desaturations in Vulnerable Ischemic Brain Tissue. Stroke 43(4): 1134-1136.

18. Damian MS, Schlosser R (2007) Bilateral near infrared spectroscopy in space-occupying middle cerebral artery stroke. Neurocrit Care 6(3): 165-173.

19. Moreau F, Yang R, Nambiar V, Demchuk AM, Dunn JF (2016) Nearinfrared measurements of brain oxygenation in stroke. Neurophotonics 3(3): 031403

20. Quaresima V, Ferrari M (2016) Functional Near-Infrared Spectroscopy (fNIRS) for Assessing Cerebral Cortex Function During Human Behavior in Natural/Social Situations: A Concise Review. Organ Res Methods 22(1): 46-68.

21. Vanzella P, Balardin JB, Furucho RA, Zimeo Morais GA, Braun Janzen T, et al. (2019) fNIRS responses in professional violinists while playing duets: Evidence for distinct leader and follower roles at the brain level. Front Psychol 10: 164.

22. Hamilton A, Pinti P, Paoletti D, Ward JA. Seeing into the Brain of an Actor with Mocap and fNIRS. In: ISWC'18: Proceedings of the 2018 ACM International Symposium on Wearable Computers. Pp. 216-217.

23. Gerrits R, Vassena E, Verguts T, Demanet J, Siugzdaite R (2019) Anticipation of a mentally effortful task recruits Dorsolateral Prefrontal Cortex: An fNIRS validation study. Neuropsychologia 123: 106-115.

24. Ayaz H, Shewokis PA, Izzetoglu M, Cakir MP, Onaral B. (2012) Tangram solved? Prefrontal cortex activation analysis during geometric problem solving. In: 2012 Annual International Conference of the IEEE Engineering in Medicine and Biology Society IEEE Engineering in Medicine and Biology Society Conference Proceedings 4724-4727.

25. Ayaz H, Shewokis PA, Curtin A, Izzetoglu M, Izzetoglu K, et al. (2011) Using MazeSuite and Functional Near Infrared Spectroscopy to Study Learning in Spatial Navigation. J Vis Exp (56): 1-13.

26. Ayaz H, Cakir MP, Izzetoglu K, et al. (2012) Monitoring Expertise Development during Simulated UAV Piloting tasks using optical Brain Imaging. In: 2012 IEEE Aerospace Conference.

27. McKendrick R, Parasuraman R, Murtza R, Formwalt A, Baccus W, et al. (2016) Into the Wild: Neuroergonomic Differentiation of Hand-Held and Augmented Reality Wearable Displays during Outdoor Navigation with Functional Near Infrared Spectroscopy. Front Hum Neurosci 10: 216.

28. Liang Z, Gu Y, Duan X, Cheng L, Liang S, et al. (2016) Design of multichannel functional near-infrared spectroscopy system with application to propofol and sevoflurane anesthesia monitoring. Neurophotonics 3(4): 045001.

29. Hirsch J, Zhang X, Noah JA, Ono Y (2017) Frontal temporal and parietal systems synchronize within and across brains during live eye-to-eye contact. Neuroimage 157: 314-330.

30. Mahoney JR, Holtzer R, Izzetoglu M, Zemon V, Verghese J, et al. (2015) The role of prefrontal cortex during postural control in Parkinsonian syndromes a functional near-infrared spectroscopy study. Brain Res 1633: $126-138$

31. Merzagora AC, Schultheis MT, Onaral B, Izzetoglu M (2011) FUNCTIONAL NEAR-INFRARED SPECTROSCOPY-BASED ASSESSMENT OF ATTENTION IMPAIRMENTS AFTER TRAUMATIC BRAIN INJURY. Journal of Innovative Optical Health Sciences 4(3): 251-260.

32. Ayaz H, Izzetoglu M, Bunce S, Heiman Patterson T, Onaral B (2007) Detecting cognitive activity related hemodynamic signal for brain computer interface using functional near infrared spectroscopy. In: 2007 3rd International IEEE/EMBS Conference on Neural Engineering 1(2): 342-345.

33. Liu Y, Ayaz H, Curtin A, Onaral B, Shewokis PA (2013) Towards a Hybrid P300-Based BCI Using Simultaneous fNIR and EEG. In: Schmorrow DD, et al. [Eds]. Foundations of Augmented Cognition. Berlin, Heidelberg: Springer Berlin Heidelberg 335-344.

34. Curtin A, Ayaz H (2018) The Age of Neuroergonomics: Towards Ubiquitous and Continuous Measurement of Brain Function with fNIRS Jpn Psychol Res 60(4): 374-386.

35. Gorm G, Terence L, Martin W (2011) Has the time come to use nearinfrared spectroscopy as a routine clinical tool in preterm infants undergoing intensive care? Philos Trans A Math Phys Eng Sci 369(1955): 4440-4451.

36. Obrig H (2014) NIRS in clinical neurology a ‘promising' tool? Neuroimage 85 Pt 1: 535-546.

37. Abdullah A, Hussain A, Khan IH (2018) A Novel Spatiotemporal Longitudinal Methodology for Predicting Obesity Using Near Infrared Spectroscopy (NIRS) Cerebral Functional Activity Data. Cognitive Computation 10(4): 591-609.

38. Atsumori H, Kiguchi M, Katura T, Funane T, Obata A, et al. (2010) Noninvasive imaging of prefrontal activation during attentiondemanding tasks performed while walking using a wearable optical topography system. J Biomed Opt 15(4): 046002.

39. McKendrick R, Mehta R, Ayaz H, Scheldrup M, Parasuraman R (2017) Prefrontal Hemodynamics of Physical Activity and Environmental Complexity During Cognitive Work. Hum Factors 59(1): 147-162.

40. Herff C, Schultz T (2016) Automatic Speech Recognition from Neural Signals: A Focused Review. Front Neurosci 10: 429.

41. Liu Y, Ayaz H (2018) Speech Recognition via fNIRS Based Brain Signals. Front Neurosci 12: 695 
42. Ayaz H, Izzetoglu M, Shewokis PA, Onaral B (2010) Sliding-window Motion Artifact Rejection for Functional Near-Infrared Spectroscopy. Conf Proc IEEE Eng Med Biol Soc 2010: 6567-6570.

43. Strangman GE, Li Z, Zhang Q (2013) Depth Sensitivity and SourceDetector Separations for Near Infrared Spectroscopy Based on the Colin27 Brain Template. PLoS One 8(8): e66319.

44. Saikia MJ, Besio WG, Mankodiya K (2019) WearLight: Toward a Wearable, Configurable Functional NIR Spectroscopy System for Noninvasive Neuroimaging. IEEE Trans Biomed Circuits Syst 13(1): 91-102.

45. Izzetoglu M, Izzetoglu K, Bunce S, Ayaz H, Devaraj A, et al. (2005) Functional near-infrared neuroimaging. IEEE Trans Neural Syst Rehabil Eng 13(2): 153-159.

46. Machado A, Cai Z, Pellegrino G, Marcotte O, Vincent T, et al. (2018) Optimal positioning of optodes on the scalp for personalized functional near-infrared spectroscopy investigations. J Neurosci Methods 309: 91108.

47. Curtis CE, DEsposito M (2003) Persistent activity in the prefrontal cortex during working memory. Trends Cogn Sci 7(9): 415-423.

48. Singh AK, Okamoto M, Dan H, Jurcak V, Dan I (2005) Spatial registration of multichannel multi-subject fNIRS data to MNI space without MRI Neuroimage 27(4): 842-851.
49. Aasted CM, Yücel MA, Cooper RJ, Dubb J, Tsuzuki D et al. (2015) Anatomical guidance for functional near-infrared spectroscopy: AtlasViewer tutorial. Neurophotonics 2(2): 020801. Cutini S, Scatturin P, Zorzi M (2011) A new method based on ICBM152 head surface for probe placement in multichannel fNIRS. Neuroimage 54(2): 919-927.

50. Cutini S, Scatturin P, Zorzi M (2011) A new method based on ICBM152 head surface for probe placement in multichannel fNIRS. Neuroimage 54(2): 919-927.

51. Tsuzuki D, Jurcak V, Singh AK, Okamoto M, Watanabe E, et al. (2007) Virtual spatial registration of stand-alone fNIRS data to MNI space. Neuroimage 34(4): 1506-1518.

52. Zimeo Morais GA, Balardin JB, Sato JR (2018) FNIRS Optodes' Location Decider (fOLD): A toolbox for probe arrangement guided by brain regions-of-interest. Sci Rep 8(1): 1-11.

53. Brigadoi S, Salvagnin D, Fischetti M, Cooper RJ (2018) Array Designer: automated optimized array design for functional near-infrared spectroscopy. Neurophotonics 5(3): 035010. 